\title{
Enhanced optical, morphological, dielectric, and conductivity properties of gold nanoparticles doped PVA/CMC blend as an application in organoelectronic devices
}

\section{R. Atta}

jouf university

Qana M. Alsulami

King Abdulaziz University

G. M. Asnag

Hajjah University

A.rajeh ( $\sim$ a.rajeh88@yahoo.com )

Amran University https://orcid.org/0000-0002-5789-0599

Original Research

Keywords:

Posted Date: February 10th, 2021

DOl: https://doi.org/10.21203/rs.3.rs-204082/v1

License: (c) (i) This work is licensed under a Creative Commons Attribution 4.0 International License.

Read Full License

Version of Record: A version of this preprint was published at Journal of Materials Science: Materials in Electronics on March 26th, 2021. See the published version at https://doi.org/10.1007/s10854-02105701-3. 
Enhanced optical, morphological, dielectric, and conductivity properties of gold nanoparticles doped PVA/CMC blend as an application in organoelectronic devices

\author{
M. R. Atta ${ }^{1,2}$, Qana M. Alsulami ${ }^{3}$, G. M. Asnag ${ }^{4}$, A. Rajeh ${ }^{5 *}$ \\ ${ }^{1}$ Physics Department, College of Science, Jouf University, P.O. Box: 2014, Sakaka, Saudi Arabia \\ ${ }^{2}$ Physics Department, Faculty of Science, Al-Azhar University, Cairo,11754, Egypt \\ ${ }^{3}$ Chemistry Department, Faculty of Science, King Abdulaziz University, P.O. Box 80203, Jeddah, 21589, \\ Saudi Arabia \\ ${ }^{4}$ Physics Department, Faculty of Education, Hajjah University, Yemen. \\ ${ }^{5}$ Physics Department, Faculty of Science, Amran University, Yemen
}

\begin{abstract}
Stevia rebaudiana plant leaves were used for biosynthesis of gold nanoparticles (AuNPs). Transmission electron microscope (TEM) images showed various shapes and sizes of AuNPs. Various amounts of AuNPs were added to polyvinyl alcohol/carboxymethylcellulose (PVA/CMC, 40/60) via the casting method. The X-ray diffraction (XRD) spectrum of pure blend shows the amorphous nature of the blend. FTIR spectra showed the interaction between PVA/CMC and AuNPs. The ultra-violet and visible (UV/VIS.) spectra showed emerge new peak of surface plasmon resonance (SPR) of AuNPs for the filled samples. SEM images showed bright spots on the sample's surface, which was attributed to AuNPs. AC conductivity exhibited enhancement after the addition of gold nanoparticles. The $\varepsilon^{\prime}$ and $\varepsilon^{\prime \prime}$ were reduced with increasing the frequency due to direction dipoles of applied electric field. Because of the mobile charges inside the polymeric backbone, higher values of $\varepsilon^{\prime}$ and $\varepsilon^{\prime \prime}$ were observed at low frequencies. The $\tan \delta$ showed increased with an increase in AuNPs concentration and at the decrease the frequency, as expected.
\end{abstract}

Keywords: Preparation of Au NPs; CMC/PVA; XRD; FT-IR; SEM; AC conductivity.

*Corresponding author: a.rajeh88@yahoo.com 


\section{I- Introduction}

Solid polymer electrolytes (SPEs) based on biopolymers have emerged as promising materials due to their potential applications in electrochemical devices[1,2] . Biopolymer materials are non-toxic, naturally abundant, renewable, bio-degradable, cost-effective and eco-friendly and can be easily obtained from natural sources like cell wall, plant,s, and animals[3,4]. Biopolymer/biodegradable polymer-based electrolytes have drawn much attention in recent years due to their eco-friendly nature and potential applications in electrochemical (EC) devices like supercapacitors, dye-sensitized solar cells (DSSCs), and batteries[5-7]. The developments of polymer electrolytes based on biopolymer are of great interest due to the presence of electron donor atoms such as $\mathrm{O}, \mathrm{N}$, etc. Donor atoms with the side chain of biopolymer interact weakly with the cations of the dissociated salt present in the polymer electrolyte[8]. Polymer nanocomposite has attained broad attention due to their high efficiency and multifunctional features obtained through the existence of just a minor quantity of the NPs inside the polymeric matrix. Polymer nanocomposites with nano-scale fillers demonstrate significant property creation at much lower loadings compared to polymer composites[8]. Importantly, the accurate selection of the host material and nano-filler is very critical for the manufacture of nanocomposites, which has a significant impact on their use and the efficiency of the devices[9]. Gold nanoparticles have received much attention because of their unique properties, such as non-cytotoxic, chemical inertness optical and electronic properties[10,11]. The development of successful green synthesis without the use of dangerous chemicals using natural reduction, capping, and stabilizing agents have attracted researchers towards green chemistry methods. The goal of adding metal nanoparticles inside polymeric matrices to develop the properties of the polymers. The structural and conductivity features of the nanocomposite will be enhanced when compared to their conventional composite[12,13]. PVA is a semi-crystalline polymer biocompatible, highly hydrophilic, nontoxic and thermoplastic polymer[14,15]. PVA is water-soluble a widely used conventional thermoplastic polymers[15]. Carboxymethyl cellulose (CMC) is mainly manufactured via reacting alkali cellulose with mono-chloroacetate or its sodium salt in an organic medium. There are many interesting properties of CMC, such as high chemical stability, strong solubility,and toxicologically harmless properties. Also is a biodegradable and 
inexpensive polymer[16,17]. In a previous work, Abdelghany et al.[18] were investigated structural, optical, and conductivity properties of CMC filled with gold nanoparticles, the optical and conductivity properties of CMC films were improved after the embedded of gold. In the present work, gold nanoparticles were used with PVA and CMC as a polymer blend[19,20]. In view of the technological useful properties of pure PVA, CMC, and AuNPs listed above, and also their use as constituents in the production of many advanced composites. In this article, aqueous solution casting was prepared using polymer blend and PVA/CMC/Au nanocomposites with varying amounts of Au NPs. The effects of $\mathrm{Au}$ nanoparticles and dielectric permittivity on dielectric polarization and electrical conductivity of prepared polymer nanocomposites of these semiconducting nanofillers have been investigated. In the development of eco-friendly, modern organelectronic devices for integrated applications, the resulting highly tunable dielectric permittivity of these composites with nanoparticle concentration confirmed their suitability as biodegradable polymer nanodielectrics.

\section{Experimental}

\subsection{Materials}

PVA was obtained by BDH-chemical Ltd Poole England Company with MW $\approx$ $17,000 \mathrm{~g} \cdot \mathrm{mol}^{-1}$. Sigma-Aldrich sealed Carboxymethyl cellulose with $\mathrm{MW} \approx 250,000$. The leaves of Stevia rebaudiana plant were supplied during July 2018 from Daqahliyah, Egypt (Figure 1). Tetrachloroauric acid $\left(\mathrm{HAuCl}_{4} 3 \mathrm{H}_{2} \mathrm{O}\right)$, with purity $99.9 \%$, was attained by Aldrich/Germany. The double-distilled water (DDW)was used to dissolve the nanocomposites components.

\subsection{Preparation of the extract of plant leaves and AuNPs green synthesis}

The leaves of Stevia rebaudiana plant were washed fully thrice with DDW. $20 \mathrm{~g}$ of washed leaves are boiled in $60 \mathrm{ml}$ of DDW in a $500 \mathrm{ml}$ Erlenmeyer flask for $45 \mathrm{~min}$ at $100{ }^{\circ} \mathrm{C}$. The leaf extract was then gathered in a separate conical flask using a normal process of filtration, i.e. Filter paper Whatman No. 40. After that, $5 \mathrm{ml}$ of Stevia rebaudiana leaves extract was added to 0.1 liliter of $1 \mathrm{mM}$ Tetrachloroauric acid in stopper-flask, and the color of the solution changes from yellow to a pinkish-red solution. This change denotes the production of AuNPs

\subsection{Nanocomposites films preparation}


Filled and unfilled PVA/CMC films were gained via casting way. PVA and CMC were dissolved in solvent separately, after that, the PVA and CMC were added with each other for $8 \mathrm{hrs}$ to get PVA/CMC blend with percentage 40/60 wt., then different concentrations $(0.0,0.05,0.10,0.20,0.40$ and 0.80$) \%$ of AuNPs were embed to the blend solution. Finally, the prepared solutions were cast into Plastic Petri dishes and leave to dry at $45^{\circ} \mathrm{C}$ for $60 \mathrm{hrs}$.

\subsection{Experimental techniques}

TEM images were gained using a JEM/1011; JEOL-Japan. X-ray diffraction pattern of the prepared samples has been recorded at room temperature using DIANO corporation-USA with $\mathrm{Cu}-\mathrm{K} \alpha$ radiation in the Bragg angle $2 \theta=10$ to 80 . Fourier transforms infrared spectra (FT-IR) (Nicolet iS10, USA) spectrophotometer measurements were performed at room temperature (RT) within the $400-4000 \mathrm{~cm}^{-1}$ range. At RT, the system of recording the absorption spectra of the samples was carried out using a UV-visible spectrophotometer (JASCO V-630-Japan) at $\lambda=190-900 \mathrm{~nm}$. The scanning electron microscope (SEM) of the samples was obtained using JEOL-SEM USA's model JSM/6510LV, with 20,000 V and a magnification of 1000 times. Conductivity measurement was carried out in the $1 \mathrm{~Hz}-$ $10 \mathrm{MHz}$ range through broadband dielectric spectroscopy (Novo control Turnkey Concept 40 System).

\section{Results and discussion}

\subsection{TEM micrograph of AuNPs}

The size and distribution of nanoparticles have been determined using TEM. Figure 2 showed the AuNPs TEM image with its corresponding histogram of size distribution. The TEM image showed that there are many forms in the AuNPs: sphere, triangular, pentagram, and irregular. The TEM micrograph for synthesized AuNPs showed nanoparticle diameter ranging between 5.5 to $30.5 \mathrm{~nm}$, this corresponds to the results of the X-ray measurement[21,22].

\section{2. XRD spectra}

Figure 3 show the XRD of AuNPs. The Figure depicted peak present at $38^{\circ}$ assigned to (111), and author peaks at $45^{\circ}, 64^{\circ}$ and $78^{\circ}$ have the miller index (200), (220) and (311) which denotes the face-centered cubic structure of AuNPs, these data correspond with data of JCPDS file no. (04-0784)[18]. Figure 4 displays the XRD pattern of PVA/CMC 
and PVA/CMC doped with different contents of gold NPs. The Figure 4 exhibit a broad peak at $7.9^{\circ}$ and other peak at $19.8^{\circ}$ which approve the amorphous feature of the PVA/CMC[23]. For the blend films filled with AuNPs we noticed that there is reduce in the two peaks intensity without change in the positions. These denote a reduce in the degree of crystallinity, as shown in Figure 4, which suggest AuNPs dispersed dramatically in the present films and caused a reduction in the degree of crystallinity, due to variation in the crosslink density, and manufacture more defects in prepared films. As a result, increasing the ionic conductivity. This shows that nanofiller adding induce interaction between gold nanoparticles and blend. These results denote that the AuNPs embed can causes structural variations in the prepared films[13,24]. The degree crystallinity (D) was obtained from the following equation[25]:

$$
D=(B / C) \times 100
$$

Where $\mathrm{B}$ represented the total area under the peaks and $\mathrm{C}$ represented the area of crystalline peaks. Table 2 exhibit a reduction in the crystallinity degree of nanocomposites, which have an agreement with FT-IR measurement and confirms the interaction between blend and nanofiller.

\section{3. FT-IR study}

FT-IR spectra have been received for the present samples, PVA/CMC blend doped with AuNPs, in the region of $400-4000 \mathrm{~cm}^{-1}$ to illustrate the interactions and complexation between blend and nanofiller as shown in Figure 5. The assignments of pure blend and nanocomposites are reported in Table 1. The spectra of blend and blend doped with different contents of gold nanoparticles in this Figure were nearly congruous to the pure blend spectrum. The influence of nanofiller on the vibrations modes was observed in terms of reducing or increasing in the intensities of the bands, broadening with Au NPs content which results due to the creation of cross-links between gold nanoparticles and functional groups in PVA/CMC blend[3] (Scheme 1). In these interactions the intensity of the bands at $1601,1415,1329$ and $1063 \mathrm{~cm}^{-1}$ increase gradually with the adding of nanoparticles content. The band at $1063 \mathrm{~cm}-1$ ascribed to glycosidic ether C-O-C stretching reduced marginally lower than pure blend, reinforcing the existence of gold NPs in the polymeric matrix[26]. These findings indicate that with blend film, the gold nanoparticles have certain interactions and complexity. It could support the gold NPs to 
transport within the chains of polymeric matrix. Thus, in the solid polymer electrolytes, these interactions play a critical role in regulating conduction behavior[27].

\section{4. UV- Vis.}

The UV-visible spectrum (Figure 6) pure Au NPs show absorption band in the visible region at $\lambda \max =526 \mathrm{~nm}$ due to surface Plasmon resonance (SPR) of AuNPs[28,29]. The appearance of this band may be related to the synthesis of AuNPs by Stevia rebaudiana plant leaves extract. Figure 7 shows UV/Vis. spectra of pure blend and its nanocomposite samples. The spectra of all prepared samples showed a sharp absorption peak nearly at $200 \mathrm{~nm}$ which was ascribed to $\Pi \rightarrow \Pi^{*}$ electronic transitions because the presence of $\mathrm{C}=\mathrm{O}$ that existed already in both CMC and PVA[14,30]. The spectra of filled samples show start emerging of a new peak at about $533 \mathrm{~nm}$ for the sample with the lowest concentration of nanofiller, which was noticed obviously in Figure 6, this peak was shifted toward a longer wavelength (red-shift)and continuously intensity increases with increased concentration of gold NPs, where this peak was appeared at about $541 \mathrm{~nm}$ for the samples of the highest concentration of nanofiller, this red-shift denotes that the sizes of nanoparticles increased with increasing its concentration via the polymeric films. This new peak was ascribed to SPR of gold NPs, which created via the reduction of $\mathrm{Au}^{3+}$ ions[31,32], which was noticed at about $526 \mathrm{~nm}$ in the spectrum of AuNPs[33].

\section{5. Morphology of samples surface (SEM)}

To investigate the influence of AuNPs content and to study the dispersion of nanoparticles in the blend matrix, the phase morphology of nanocomposites samples were investigated via SEM. The SEM micrographs of the sample's surface are present in Figure 8. For the pristine film, as shown in Figure 8(a), the film's surface was uniform surface morphology, also a rather smooth surface. After added AuNPs to the polymeric blend, as depicted in Figure 8(b-f), bright spots and spots groups appeared on the surface of the films without any aggregation except in the highest concentration[34,35]. The bright spots increase as nanofiller concentration increase which denotes that these spots perhaps are of gold nanoparticles and propose the occurrence of a homogeneous growth mechanism[36]. The occurred variations at the filled sample's surface denote proved the complexation and interaction compatibility between the gold nanoparticles and the pure 
PVA/CMC. The SEM micrographs were a good approval with the previous measurements.

\section{6. Electrical conductivity:}

The AC conductivity of all samples of PVA/CMC-AuNPs in the frequency $(f)$ range between $0.1 \mathrm{~Hz}$ to $2 \times 10^{7} \mathrm{~Hz}$ at room temperature, which are showed in Figure 9, the AC conductivity were calculated by equation follow[11,37]:

$$
\sigma=\frac{L}{R A}
$$


Where $\mathrm{R}$ represents the resistance, while $\mathrm{L}$ is samples thickness and A represents the electrode's area. In general, the behavior of pure sample ac conductivity shows that the frequency dependence due to the polymeric nature is insulating materials but the ac conductivity of the nanocomposites films after addition the AuNPs was enhanced several orders of magnitude. At the highest concentration (0.8 wt.\%) of the AuNPs in the polymeric matrix, the highest value of AC conductivity. This improved was due to the electronics and impurities contributions arising from the AuNPs, also, due to the increment for free volume around the polymeric networks reasons the mobility for polarons and/or bipolrons [38,39]. Also, at the increasing of AuNPs concentration, the molecules begin to bridge the gap separated between two localized states and the potential barriers between them, thereby expediting of the transfer of charge carriers[40,41]. An improvment of the ac conductivity for all nanocomposite samples compared to pure PVA/CMC is witnessed (see Fig.9). Among all prepared samples, Au/PVA/CMC films display the highest conductivity at a concentration (0.8 wt.\%) of AuNPs. These findings align with the results of XRD, in which the adding of AuNPs into the PVA/CMC blend increases the disorder degree (see Fig. 3) of the Au/PVA/CMC films and therefore, promotes the ion charges mobility within the nanocomposites, leading to an enhancement of conductivity[41,42]. In addition, the findings of the optical band-gap values, in which the Au/PVA/CMC (0.8wt.\%) has the lowest direct and indirect band-gap (3.6 eV and 2.9 $\mathrm{eV}$ ), support these findings. These ac conductivity values are higher than those that other reports have achieved[43,44].

\section{7. Dielectric properties}

The dielectric constant $\left(\varepsilon^{\prime}\right)$ and dielectric loss $\left(\varepsilon^{\prime \prime}\right)$ was calculated using the equation [45]:

$$
\varepsilon^{\prime}=\mathrm{C} \mathrm{d} / \varepsilon_{\mathrm{o}} \mathrm{A}
$$

Where $\mathrm{d}$ is the film thickness, $\mathrm{C}$ is the capacitance, $\mathrm{A}$ is the cross-sectional area of the electrode, and $\varepsilon_{0}$ represented free space-permittivity $\left(\varepsilon_{o}=8.85 \times 10^{-12}\right.$ F.m $\left.\mathrm{m}^{-1}\right)$.

$$
\varepsilon^{\prime \prime}=\sigma / \omega \varepsilon_{0}
$$

Where $\omega$ is represent angular frequency. 
Figure 10(a,b) exhibited the $\varepsilon^{\prime}$ and the $\varepsilon^{\prime \prime}$ versus on frequency of the all prepared films are high, due to the direction of the dipole via samples to orient them in direction of the applied field. The reducing trend of $\varepsilon^{\prime}$ and the $\varepsilon^{\prime \prime}$ appears almost stable at higher frequencies compared with lower frequencies. This trend was assigned to dipoles orientation, causing difficulty to rotate dipoles at high frequencies. Moreover, at low frequencies, the $\varepsilon^{\prime}$ and the $\varepsilon^{\prime \prime}$ values were higher due to the effect of interfacial of the samples and the effect of the electrode[46]. Also, the $\varepsilon^{\prime}$ and $\varepsilon^{\prime \prime}$ increased gradually with AuNPs concentration denotes that the higher charge carrying capacity of loaded samples compared to pure sample. In terms of electrical conductivity, the higher value of the dielectric loss at higher concentrations of AuNPs can be understood. Furthermore, the mobile charges (polarons/bipolrons) that belong to conducting polymer and free charges increase as AuNPs concentration increases, cause influence lower value of $\varepsilon^{\prime}$ and $\varepsilon^{\prime \prime}$ at higher frequencies[47].

The loss tangent $(\tan \delta)$ is another significant parameter that provides insight into the charge carrier's number available for the conduction mechanism[48]. The $\tan \delta$ is defined as the ratio between energy loss and energy stored[49]. The changes in tangent loss are obtained as[50]:

$$
\tan \delta=\varepsilon^{\prime \prime} / \varepsilon^{\prime}
$$

The $\tan \delta$ versus frequency graph given in Fig. 11 confirms that $\tan \delta$ was substantially higher in these nanocomposite films at $30 \mathrm{~Hz}$ as compared to the pure PVA/CMC film values, which was certainly due to the interfacial polarization effect contribution. However, with an increasing frequency starting from $30 \mathrm{~Hz}$, these losses decreased sharply and, over 1 $\mathrm{kHz}$, the $\tan \delta$ became almost frequency independent with relatively very low values. Moreover, in the experimental frequency range for these polymer nanocomposite materials, there was no relaxation process corresponding to the interfacial polarization effect, this is consistent with a previous study[51].

\section{Conclusions}

The AuNPs were created using Stevia rebaudiana leafs extract, which was confirmed by occurrence the SPR of NPs in UV-Vis. spectrum and from the TEM micrograph. The nanocomposite of PVA/CMC-AuNPs was reported and investigated by various 
measurements. XRD measurement of nanocomposites films exhibited the decrease in the crystallinity after embedded the AuNPs due to the change in the crosslink density of PVA/CMC. The most obvious complexation between polymer blend and dopant occurred at wavenumber 900 to $2200 \mathrm{~cm}-1$ and 2700 to $4000 \mathrm{~cm}-1$ based on the FTIR study, where the peaks observed are assigned to $\mathrm{CO},-\mathrm{OH}, \mathrm{COO}$ and AuNPs. The absorbance in the UV-Vis range has increased and the optical energy band gap has reduced. The SEM images revealed white granules on the films surfaces which proved the presence of AuNPs in the nanocomposites films. The increase in ionic conductivity after the adding of AuNPs is associated with an increase in the coefficient of ion mobility and diffusion. The highest ionic conductivity was obtained at room temperature for the blend doped with 0.8 wt. $\%$ of AuNPs. The increase in the film conductivity is proved by XRD, SEM, and UV-Vis. characterization. The $\varepsilon^{\prime}$ and the $\varepsilon^{\prime \prime}$ vs. frequency is plotted at various contents and at room temperature. Based on these findings, it is believed that nanocomposites based on PVA/CMC doped with AuNPs could suggest a new insight into their potential use in energy storage systems.

\section{References:}

[1] C. Yang, S. Lin, Alkaline composite PEO - PVA - glass-fibre-mat polymer electrolyte for Zn - air battery, 112 (2002) 497-503.

[2] R. Kandulna, R.B. Choudhary, Concentration-dependent behaviors of ZnO-reinforced PVA-ZnO nanocomposites as electron transport materials for OLED application, Polym. Bull. 75 (2018) 3089-3107. https://doi.org/10.1007/s00289-017-2186-9.

[3] A.M. Hezma, A. Rajeh, M.A. Mannaa, An insight into the effect of zinc oxide nanoparticles on the structural, thermal, mechanical properties and antimicrobial activity of Cs/PVA composite, Colloids Surfaces A Physicochem. Eng. Asp. 581 (2019) 123821. https://doi.org/10.1016/J.COLSURFA.2019.123821.

[4] A.R. A. M. Hezma, Spectroscopic and Drug Delivery studies of Moxifloxacin Loaded Polylactic Acid/Polycaprolacton Polymeric Matrix for Medical Application, Res. J. Pharm. Biol. Chem. Sci. 8 (2017) 1163-1170.

[5] T. Bourtoom, M.S. Chinnan, Preparation and properties of rice starch-chitosan blend biodegradable film, LWT - Food Sci. Technol. 41 (2008) 1633-1641. https://doi.org/10.1016/J.LWT.2007.10.014.

[6] X. Ma, P.R. Chang, J. Yu, Properties of biodegradable thermoplastic pea starch/carboxymethyl cellulose and pea starch/microcrystalline cellulose composites, Carbohydr. Polym. 72 (2008) 369-375. https://doi.org/10.1016/J.CARBPOL.2007.09.002. 
[7] A. De Campos, J.C. Marconato, S.M. Martins-franchetti, Biodegradation of Blend Films PVA / PVC, PVA / PCL in Soil and Soil with Landfill Leachate, Brazilian Arch. Biol. Technol. 54 (2011) 1367-1378. https://doi.org/10.1590/S1516-89132011000600024.

[8] 1. P. K. Varshney, S. Gupta , Natural polymer-based electrolytes for electrochemical devices: A review. Ionics (2011) 17479-483, (n.d.).

[9] M.M. Abutalib, Insights into the structural, optical, thermal, dielectric, and electrical properties of PMMA/PANI loaded with graphene oxide nanoparticles, Phys. B Condens. Matter. 552 (2019) 19-29. https://doi.org/10.1016/J.PHYSB.2018.09.034.

[10] L.H. Gaabour, Spectroscopic and thermal analysis of polyacrylamide/chitosan (PAM/CS) blend loaded by gold nanoparticles, Results Phys. 7 (2017) 2153-2158. https://doi.org/10.1016/J.RINP.2017.06.027.

[11] A.M. Abdelghany, A.H. Oraby, M.O. Farea, Influence of green synthesized gold nanoparticles on the structural, optical, electrical and dielectric properties of (PVP/SA) blend, Phys. B Condens. Matter. $560 \quad$ (2019) 162-173. https://doi.org/10.1016/J.PHYSB.2019.02.029.

[12] H.M. Ragab, A. Rajeh, Structural, thermal, optical and conductive properties of PAM/PVA polymer composite doped with Ag nanoparticles for electrochemical application. J Mater Sci: Mater Electron 31(2020) 16780-16792., (n.d.).

[13] E.M. Abdelrazek, I.S. Elashmawi, A.M. Hezma, A. Rajeh, M. Kamal, Effect of an encapsulate carbon nanotubes (CNTs) on structural and electrical properties of PU/PVC nanocomposites, Phys. B Condens. Matter. 502 (2016) 48-55. https://doi.org/10.1016/j.physb.2016.08.040.

[14] I.S. Elashmawi, E.M. Abdelrazek, A.M. Hezma, A. Rajeh, Modification and development of electrical and magnetic properties of PVA/PEO incorporated with $\mathrm{MnCl} 2$, Phys. B Condens. Matter. 434 (2014) 57-63. https://doi.org/10.1016/j.physb.2013.10.038.

[15] A. Abdelrazek, E. M., Elashmawi, I. S., Hezma, A., Rajeh, The Accessibility of Change of the Structural, Morphological and Thermal Properties by Intermolecular Hydrogen Bonding in PEO/PVA Blend Containing MnCl2, Int. J. Mod. App. Phys. 2 (2012) 83-96.

[16] N.H. Ahmad, M.I.N. Isa, Characterization of un-plasticized and propylene carbonate plasticized carboxymethyl cellulose doped ammonium chloride solid biopolymer $\begin{array}{lllll}\text { electrolytes, } & \text { Carbohydr. } & \text { Polym. } & 137 & \text { (2016) }\end{array}$ https://doi.org/10.1016/J.CARBPOL.2015.10.092.

[17] A. Rajeh, M.A. Morsi, I.S. Elashmawi, Enhancement of spectroscopic, thermal, electrical and morphological properties of polyethylene oxide/carboxymethyl cellulose blends: $\begin{array}{lllll}\text { Combined } & \text { FT-IR/DFT, } & \text { Vacuum. } & 159 & \text { (2019) }\end{array}$ https://doi.org/10.1016/J.VACUUM.2018.10.066.

[18] A. M. Abdelghany, A. H. Oraby, G. M. Asnag, Journal of Molecular Structure, 1180 
(2019) 15-25, (n.d.).

[19] E. M. Abdel Bary, A. Fekri, Y. A. Soliman, A. N. Harmal, Polymer Bulletin, 75 (2018) 4977-4997., (n.d.).

[20] A. Singh, S. Mittal, R. Shrivastav, S. Dass, J. Srivastava, Digest Journal of Nanomaterials and Biostructures, 7 (2012) 1157-1163. , (n.d.).

[21] M.M. Abutalib, Effect of zinc oxide nanorods on the structural, thermal, dielectric and electrical properties of polyvinyl alcohol/carboxymethyle cellulose composites, Phys. B Condens. Matter. 557 (2019) 108-116. https://doi.org/10.1016/J.PHYSB.2019.01.018.

[22] S. AL-Thabaiti, M. Malik, A. Al-Youbi, Z. Khan, J. Hussain, International Journal of Electrochemical Science, 8 (2013) 204-218., (n.d.).

[23] A. Rajeh, M.A. Morsi, I.S. Elashmawi, Enhancement of spectroscopic, thermal, electrical and morphological properties of polyethylene oxide/carboxymethyl cellulose blends: $\begin{array}{lllll}\text { Combined } & \text { FT-IR/DFT, } & \text { Vacuum. } & 159 & \text { (2019) 430-440. }\end{array}$ https://doi.org/10.1016/J.VACUUM.2018.10.066.

[24] Sunardi, N. M. Febriani, A. B.Junaidi, American Institute of Physics, (2017) 1-8., (n.d.).

[25] A.M. Hezma, I.S. Elashmawi, A. Rajeh, M. Kamal, Change Spectroscopic, thermal and mechanical studies of PU/PVC blends, Phys. B Condens. Matter. 495 (2016) 4-10. https://doi.org/10.1016/j.physb.2016.04.043.

[26] C. Basavaraja, J. Kim, D. Huh, Materials Science and Engineering B, 178 (2013) 167-173., (n.d.).

[27] M.A. Morsi, S.A. El-Khodary, A. Rajeh, Enhancement of the optical, thermal and electrical properties of PEO/PAM:Li polymer electrolyte films doped with $\mathrm{Ag}$ nanoparticles, Phys. B Condens. Matter. 539 (2018) 88-96. https://doi.org/10.1016/J.PHYSB.2018.04.009.

[28] A. Hebeish, M. Hashem, M.Abd El-Hady, S. Sharaf, Carbohydrate Polymers, 92 (2013) 407-413., (n.d.).

[29] I. Jahit, N. Nazmi, M. Isa, N. Sarbon, International Food Research Journal, 3 (2016) 10681074., (n.d.).

[30] M.M. Abutalib, A. Rajeh, Influence of MWCNTs/Li-doped TiO2 nanoparticles on the structural, thermal, electrical and mechanical properties of poly (ethylene oxide)/poly (methylmethacrylate) composite, J. Organomet. Chem. 918 (2020) 121309. https://doi.org/10.1016/J.JORGANCHEM.2020.121309.

[31] M. Pattanayak, T. Muralikrishnan, P. Nayak, World Journal of Nano Science \& Technology, 2 (2014) 52-58, (n.d.).

[32] S. V. Kumar, S. Karpagambigai, P. J. Rosy, S. Rajeshkumar, Mechanics, Materials Science 
\& Engineering, (2017) 1-8, (n.d.).

[33] A.M. Abdelghany, E.M. Abdelrazek, S.I. Badr, M.A. Morsi, Effect of gamma-irradiation on (PEO/PVP)/Au nanocomposite: Materials for electrochemical and optical applications, Mater. Des. 97 (2016) 532-543. https://doi.org/10.1016/j.matdes.2016.02.082.

[34] G. Patel, M. Sureshkumar, P. Patel, Soft, Scientific Research Publishing, 4 (2015) 9-24, (n.d.).

[35] X. Qi, S. Liu, F. Chu, S. Pang, Y. Liang, Y. Guan, F. Peng, R. Sun, Materials (2015) 1-12, (n.d.).

[36] N. Haleem, M. Arshad, M. Shahid, M. A. Tahir, Carbohydrate Polymers 113 (2014) 249255 , (n.d.).

[37] M.O. Farea, A.M. Abdelghany, M.S. Meikhail, A.H. Oraby, Effect of cesium bromide on the structural, optical, thermal and electrical properties of polyvinyl alcohol and polyethylene oxide, J. Mater. Res. Technol. (2019). https://doi.org/10.1016/J.JMRT.2019.11.078.

[38] M.M. Abutalib, A. Rajeh, Preparation and characterization of polyaniline/sodium alginatedoped $\mathrm{TiO} 2$ nanoparticles with promising mechanical and electrical properties and antimicrobial activity for food packaging applications, J. Mater. Sci. Mater. Electron. 31 (2020) 1-13. https://doi.org/10.1007/s10854-020-03483-8.

[39] M.M. Abutalib, A. Rajeh, Influence of $\mathrm{Fe} 3 \mathrm{O} 4$ nanoparticles on the optical, magnetic and electrical properties of PMMA/PEO composites: Combined FT-IR/DFT for electrochemical applications, J. Organomet. Chem. $920 \quad$ (2020) 121348. https://doi.org/10.1016/J.JORGANCHEM.2020.121348.

[40] M.M. Abutalib, A. Rajeh, Preparation and characterization of polyaniline/sodium alginatedoped $\mathrm{TiO} 2$ nanoparticles with promising mechanical and electrical properties and antimicrobial activity for food packaging applications, J. Mater. Sci. Mater. Electron. 31 (2020) 9430-9442. https://doi.org/10.1007/s10854-020-03483-8.

[41] M.A. Morsi, A. Rajeh, A.A. Al-Muntaser, Reinforcement of the optical, thermal and electrical properties of PEO based on MWCNTs/Au hybrid fillers: Nanodielectric materials for organoelectronic devices, Compos. Part B Eng. 173 (2019). https://doi.org/10.1016/j.compositesb.2019.106957.

[42] A. Rajeh, H.M. Ragab, M.M. Abutalib, Co doped ZnO reinforced PEMA/PMMA composite: Structural, thermal, dielectric and electrical properties for electrochemical applications, J. Mol. Struct. 1217 (2020). https://doi.org/10.1016/j.molstruc.2020.128447.

[43] W. Tongdeesoontorn, L.Mauer, S. Wongruong, P. Sriburi, P. Rachtanapun, Chemistry Central Journal, (2011) 1-8, (n.d.).

[44] E. M. Abdelrazek, I. Elashmawi, H. Ragab, Physica B, 403 (2008) 3097-3104., (n.d.). 
[45] A.S. Ayesh, Electrical and optical characterization of PMMA doped with Y0.0025Si0.025Ba0.9725 (Ti(0.9)Sn0.1)O3 ceramic, Chinese J. Polym. Sci. 28 (2010) 537-546. https://doi.org/10.1007/s10118-010-9086-x.

[46] H. Peng, G. Ma, W. Ying, A. Wang, H. Huang, Z. Lei, Journal of Power Sources, 211 (2012) 40-45, (n.d.).

[47] Q. A. Alsulami, Structural, dielectric, and magnetic studies based on MWCNTs/NiFe2O4/ZnO nanoparticles dispersed in polymer PVA/PEO for electromagnetic applications. J Mater Sci: Mater Electron (2021). https://doi.org/10.1007/s10854-020-05043-6, (n.d.).

[48] M. M. Abutalib, A. Rajeh, "Enhanced structural, electrical, mechanical properties and antibacterial activity of $\mathrm{Cs} / \mathrm{PEO}$ doped mixed nanoparticles $(\mathrm{Ag} / \mathrm{TiO} 2)$ for food packaging applications." Polymer Testing 93 (2020) 107013., (n.d.).

[49] K.N. Kumar, M. Kang, K. Sivaiah, M. Ravi, Y.C. Ratnakaram, Enhanced electrical properties of polyethylene oxide (PEO) + polyvinylpyrrolidone (PVP):Li+ blended polymer electrolyte films with addition of Ag nanofiller, Ionics (Kiel). 22 (2016) 815-825. https://doi.org/10.1007/s11581-015-1599-4.

[50] O. Pravakar, T. Siddaiah, P.V.R.K. Ramacharyulu, N.O. Gopal, C. Ramu, H. Nagabhushana, Spectroscopic, thermal, structural and electrical studies on VO2+ ions doped PVA/MAA:EA polymer blend films, J. Sci. Adv. Mater. Devices. 4 (2019) 267275. https://doi.org/10.1016/J.JSAMD.2019.03.004.

[51] G.M. Asnag, A.H. Oraby, A.M. Abdelghany, Green synthesis of gold nanoparticles and its effect on the optical, thermal and electrical properties of carboxymethyl cellulose, $\begin{array}{lllll}\text { Compos. Part } & B & \text { Eng. } & 172 & \text { (2019) 436-446. }\end{array}$ https://doi.org/10.1016/J.COMPOSITESB.2019.05.044. 
Figures

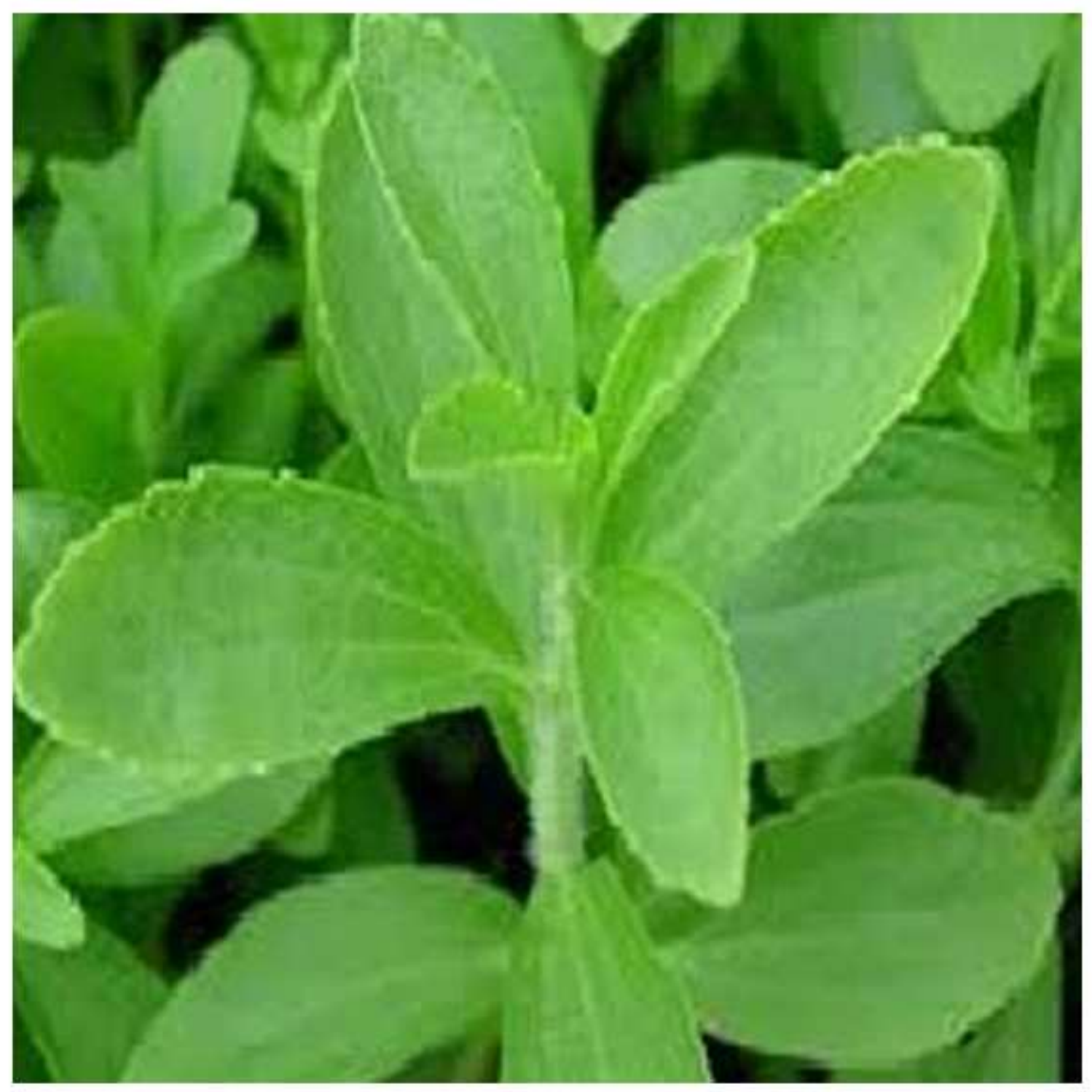

Figure 1

The Stevia rebaudiana plant. 

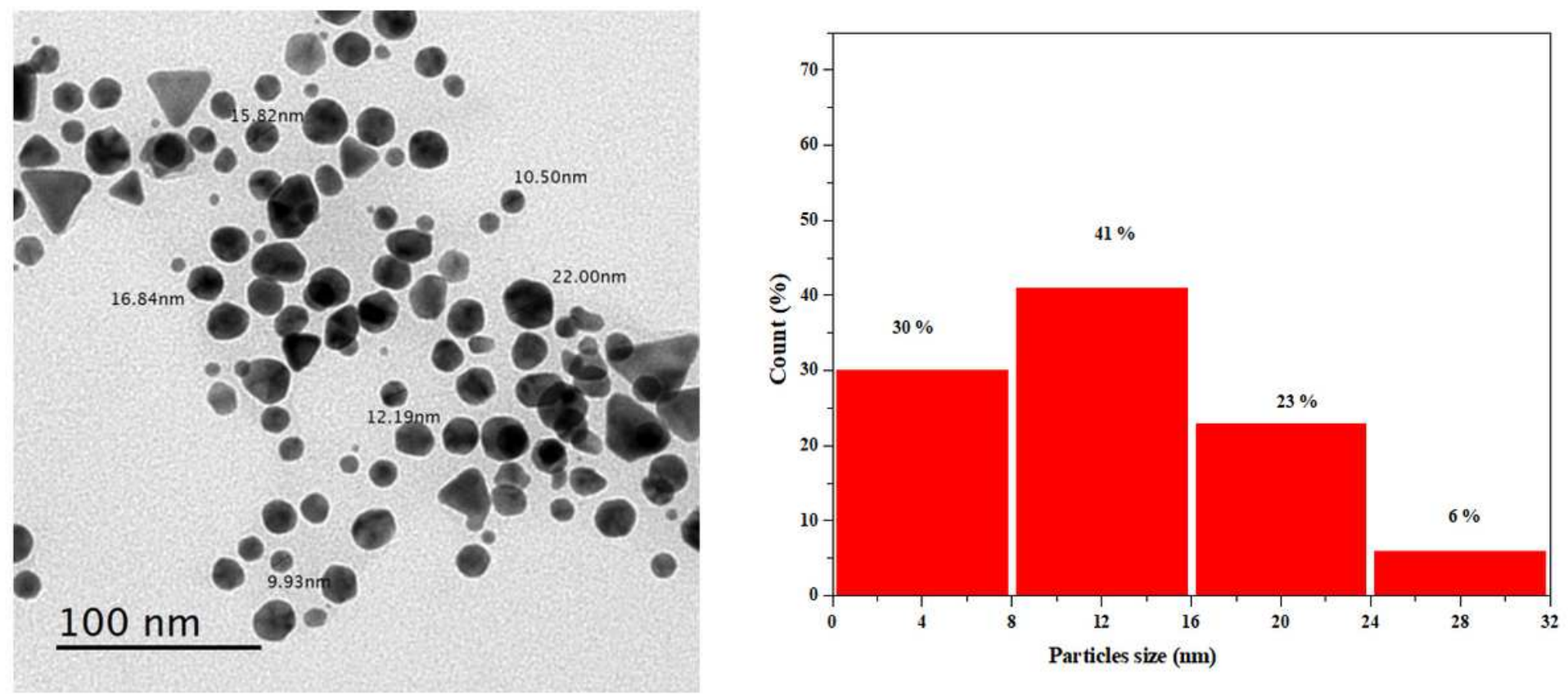

Figure 2

The TEM micrograph and the histogram of size distribution green synthesized AuNPs.

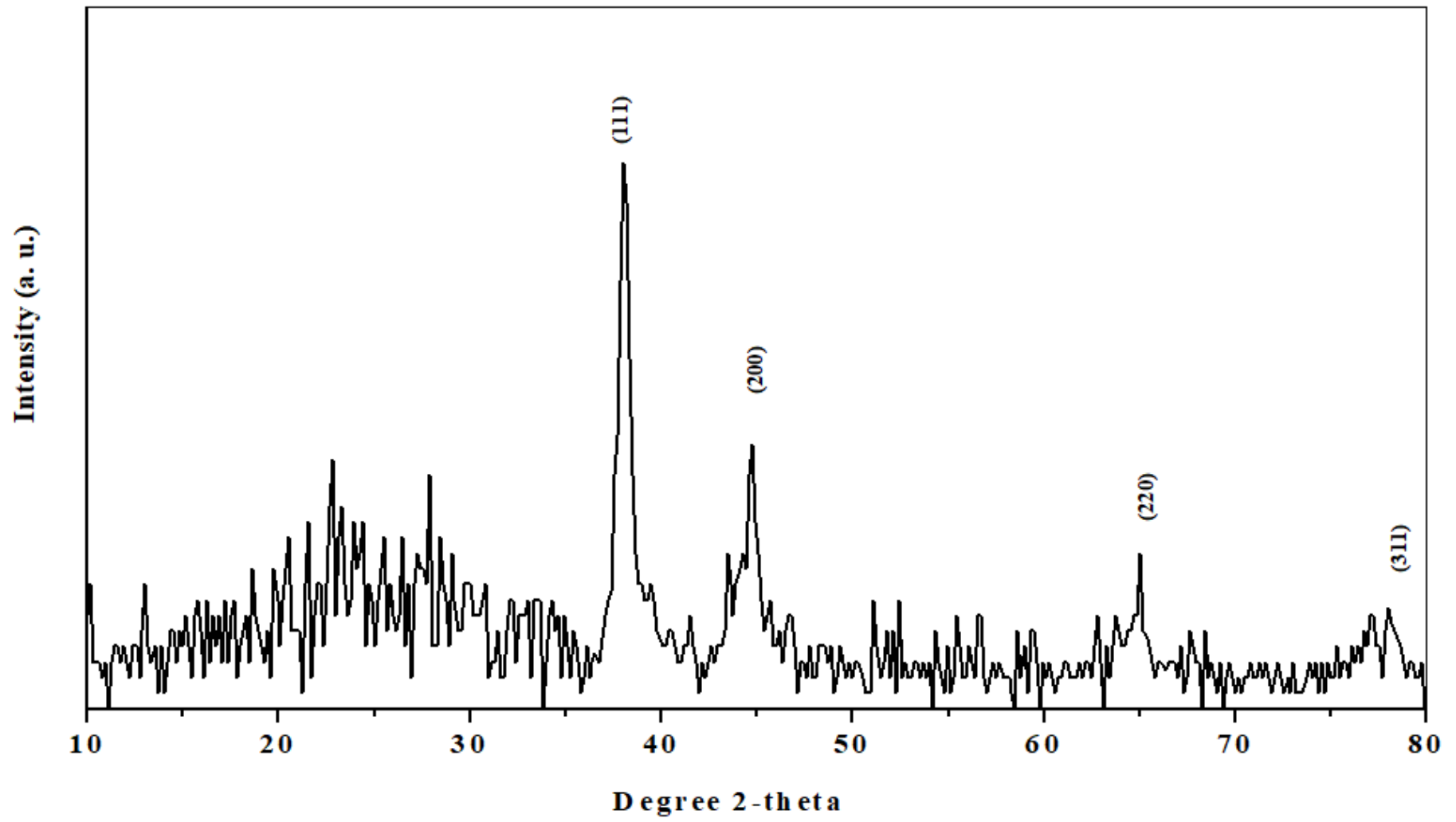

Figure 3

XRD pattern for AuNPs. 


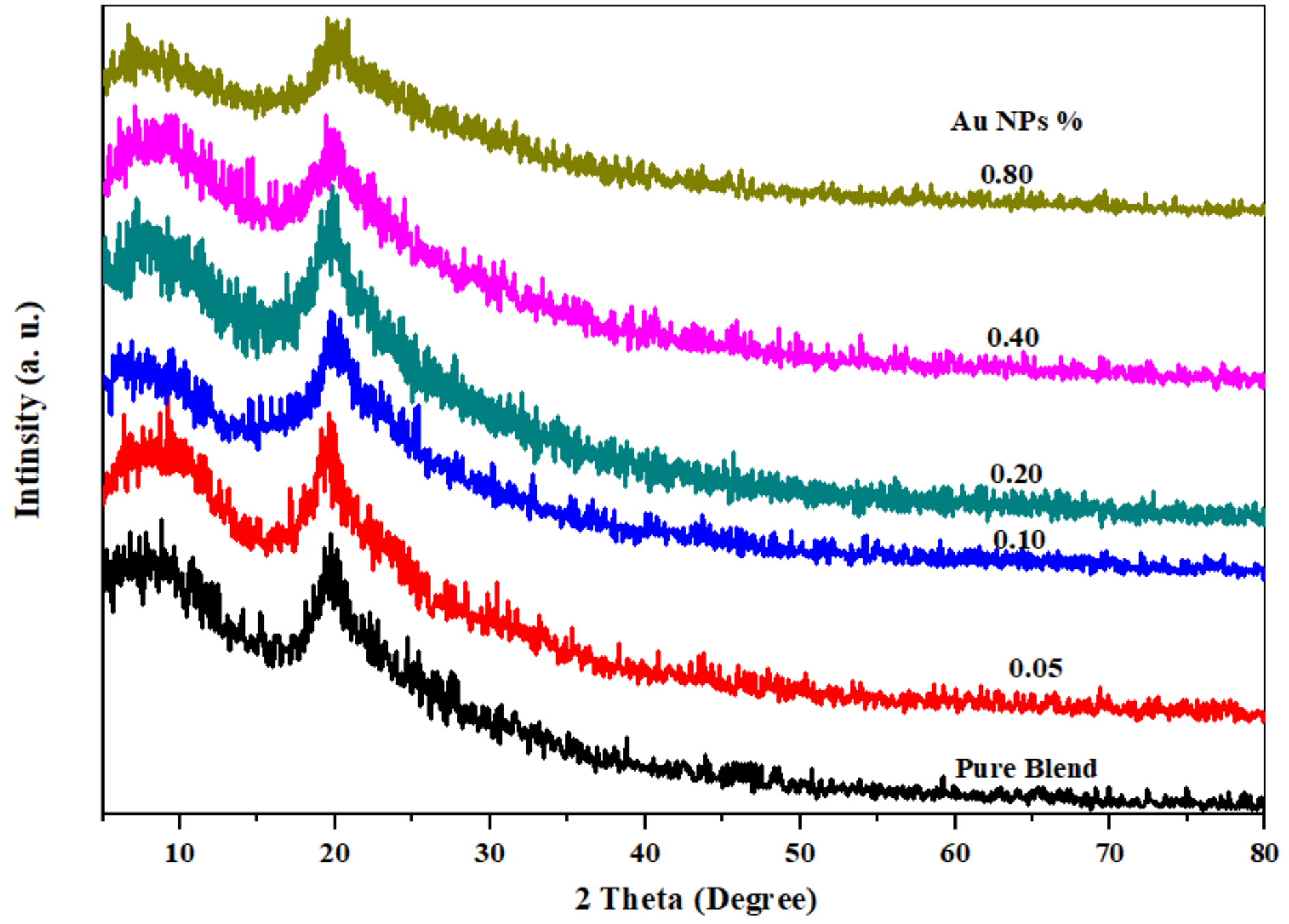

Figure 4

Pure PVA/CMC blend and filled samples with various concentrations of AuNPs. 


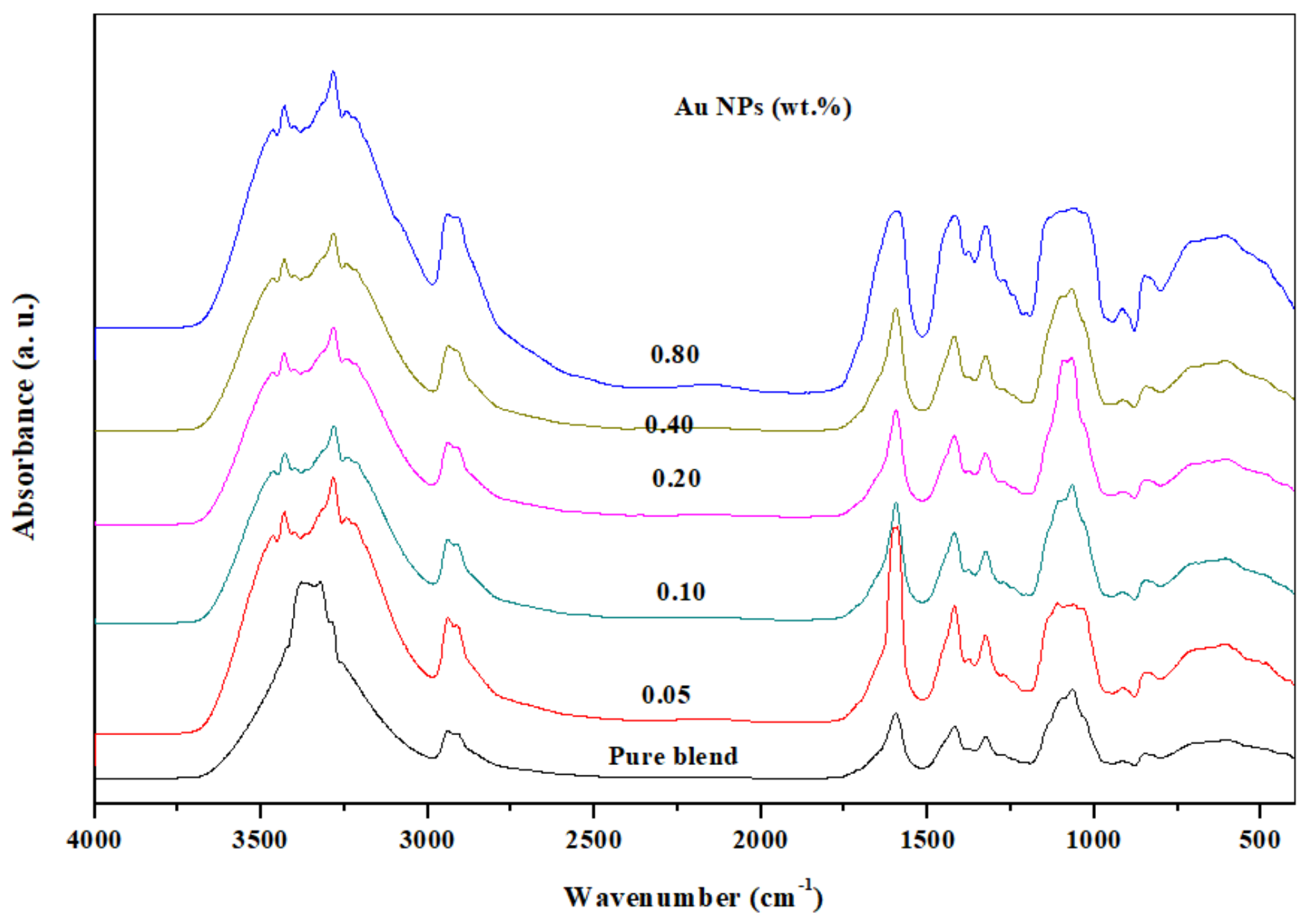

Figure 5

FT-IR spectra of pure blend with different concentration of gold nanoparticles. 


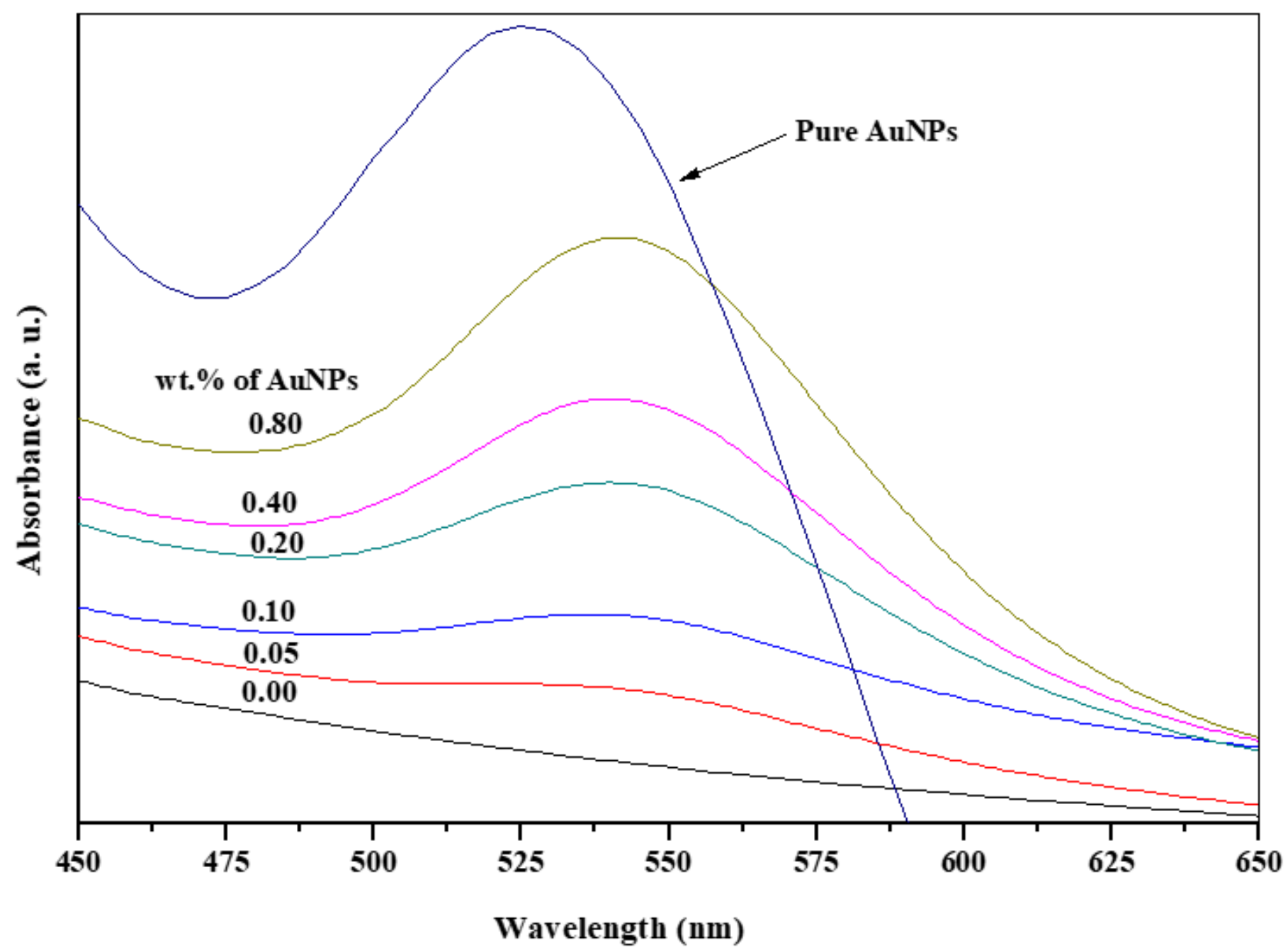

Figure 6

UV/VIS. spectra of pure AuNPs and PVA/CMC-AuNPs nanocomposites in the wavelength of $450-650 \mathrm{~nm}$. 


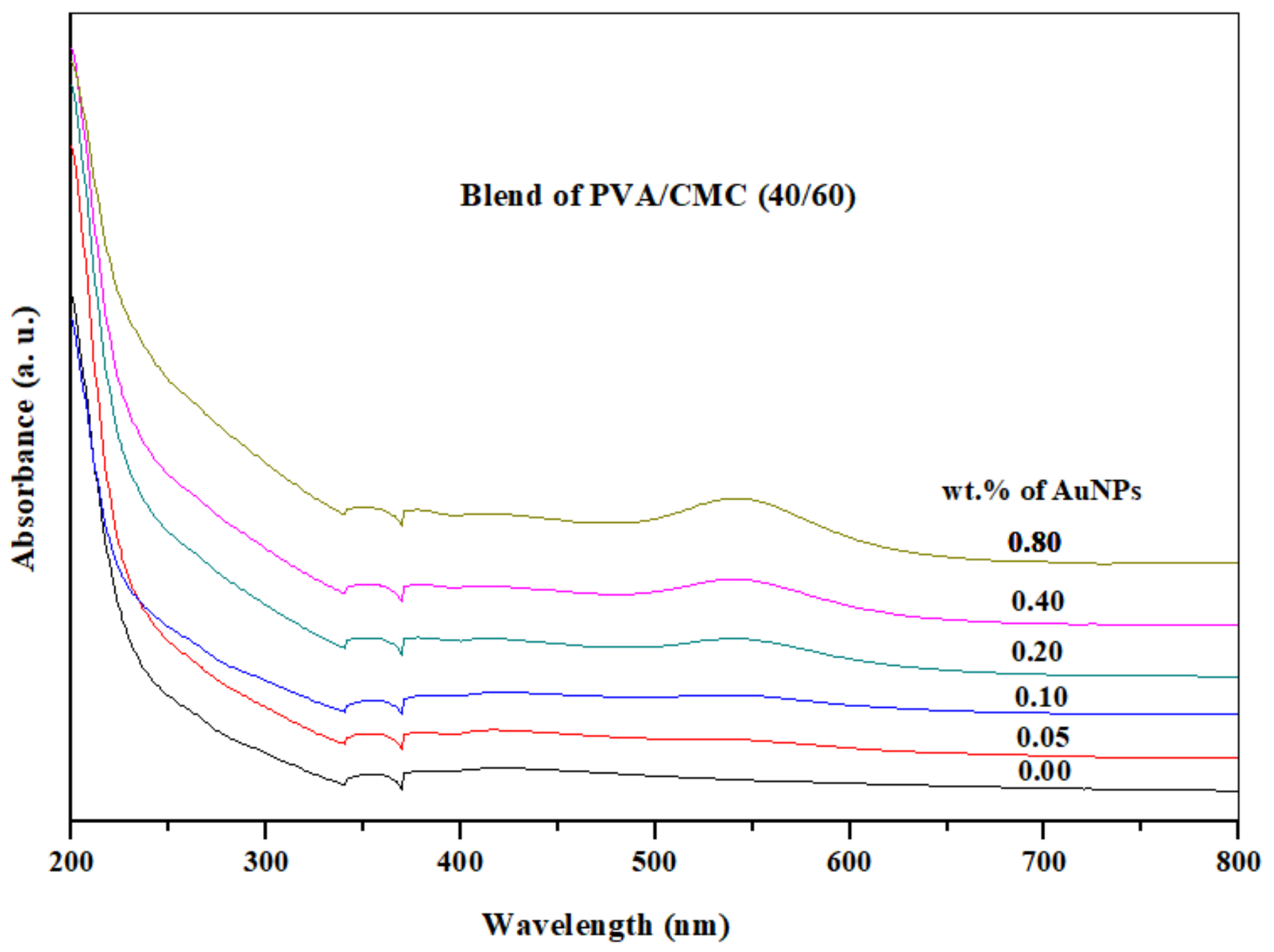

Figure 7

UV/VIS. spectra of PVA/CMC-AuNPs nanocomposites. 


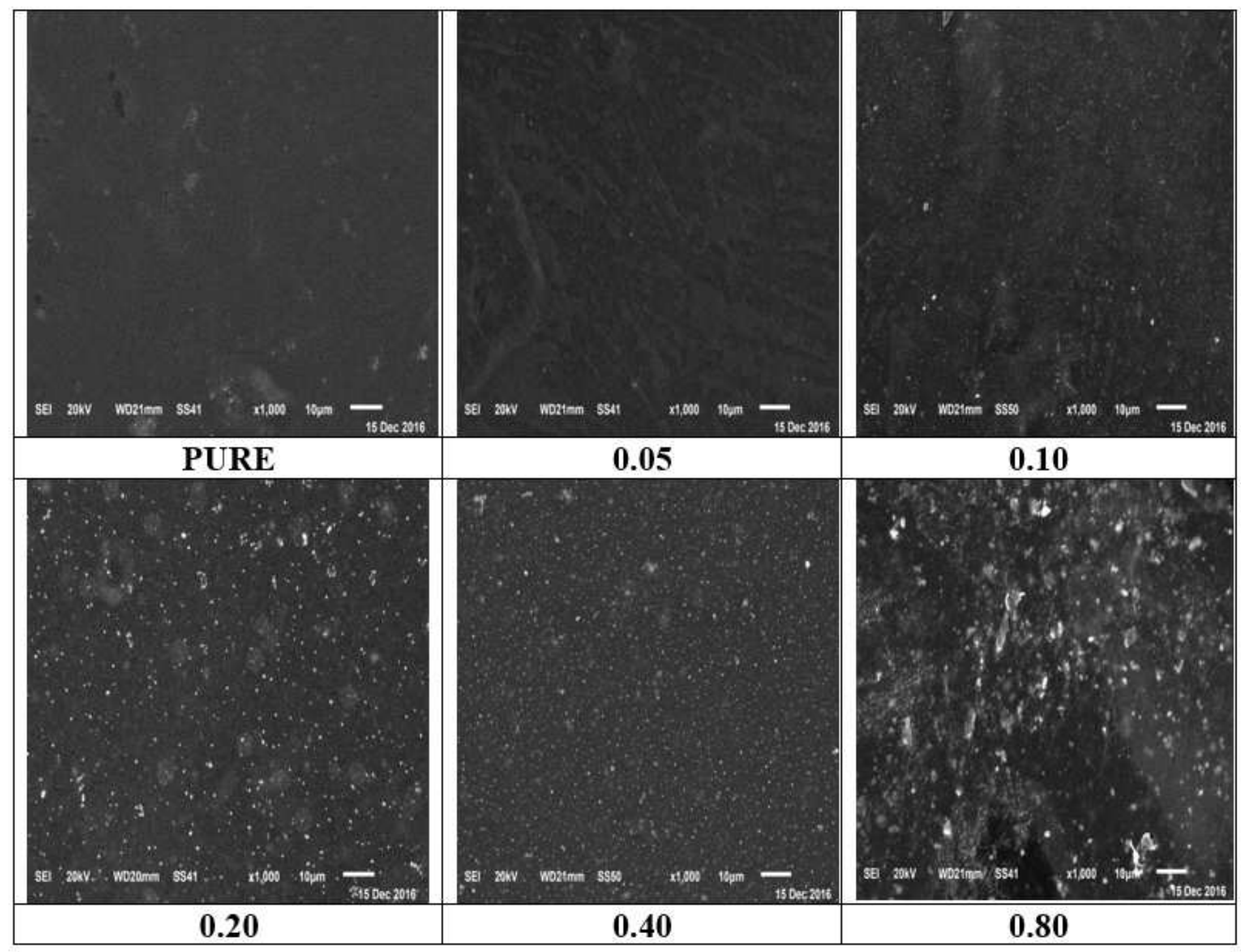

Figure 8

The SEM images of the samples surfaces of pure blend and its complexes doped with various concentrations AuNPs at magnification 1000 times. 


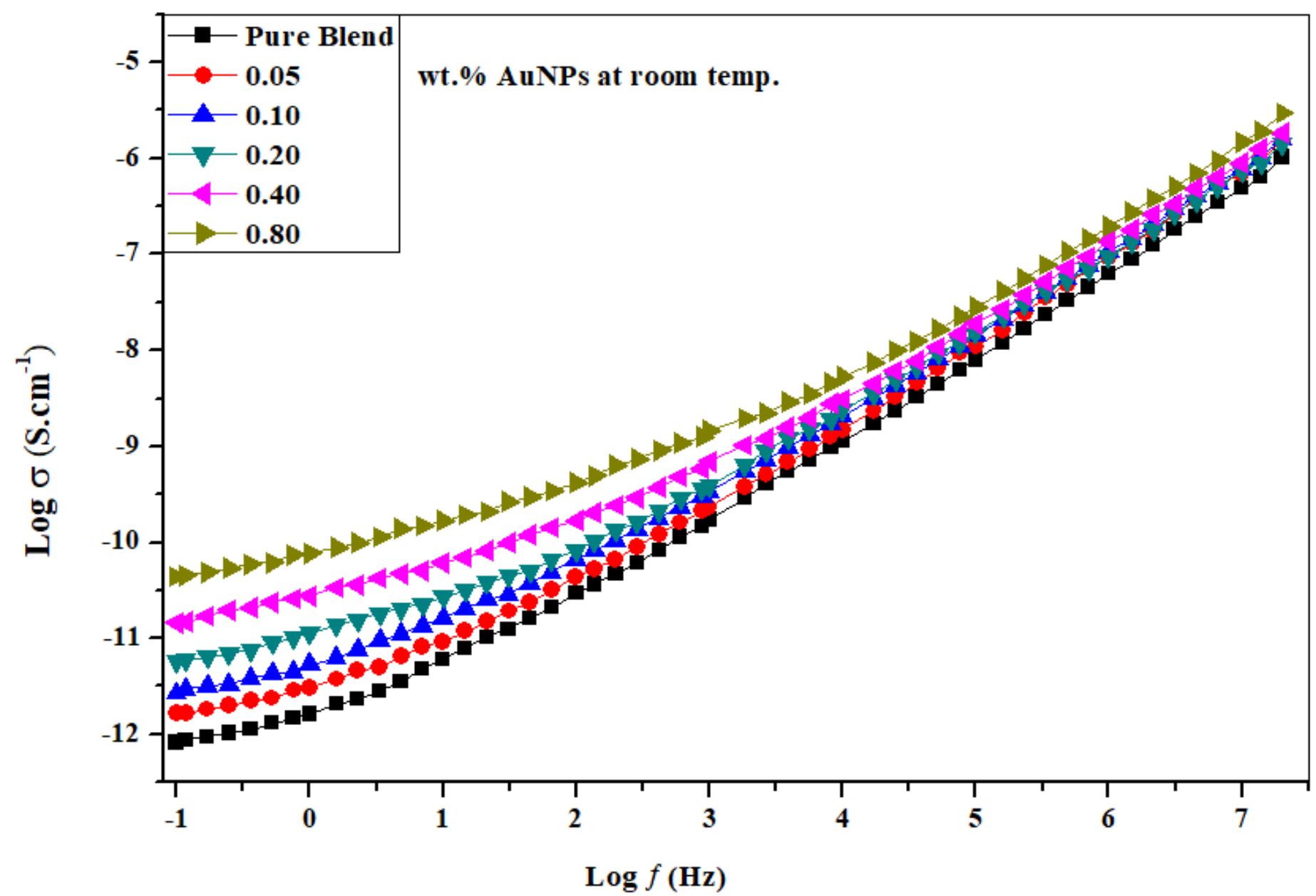

Figure 9

The ac conductivity $(\log \sigma)$ versus $\log f$ of PVA/CMC with different concentrations of AuNPs at room temperatures.
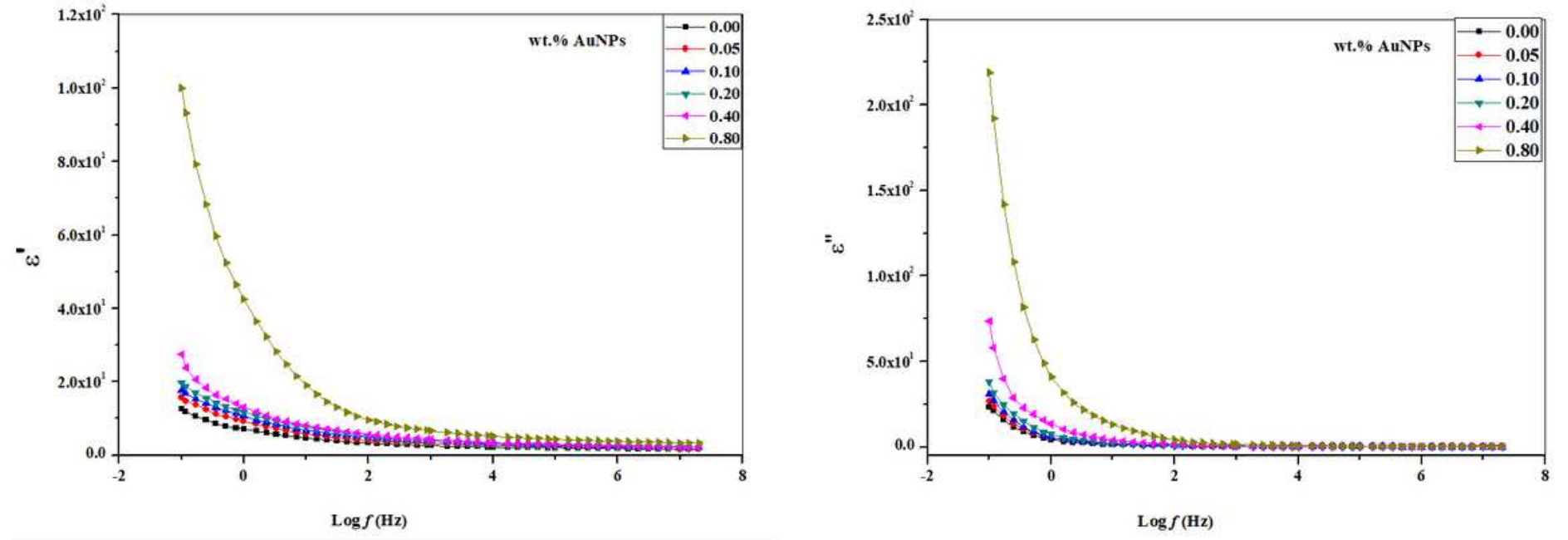

Figure 10

Variation of $\varepsilon^{\prime}$ and $\varepsilon^{\prime \prime}$ with respect to frequency of nanocomposites samples at room temperatures. 


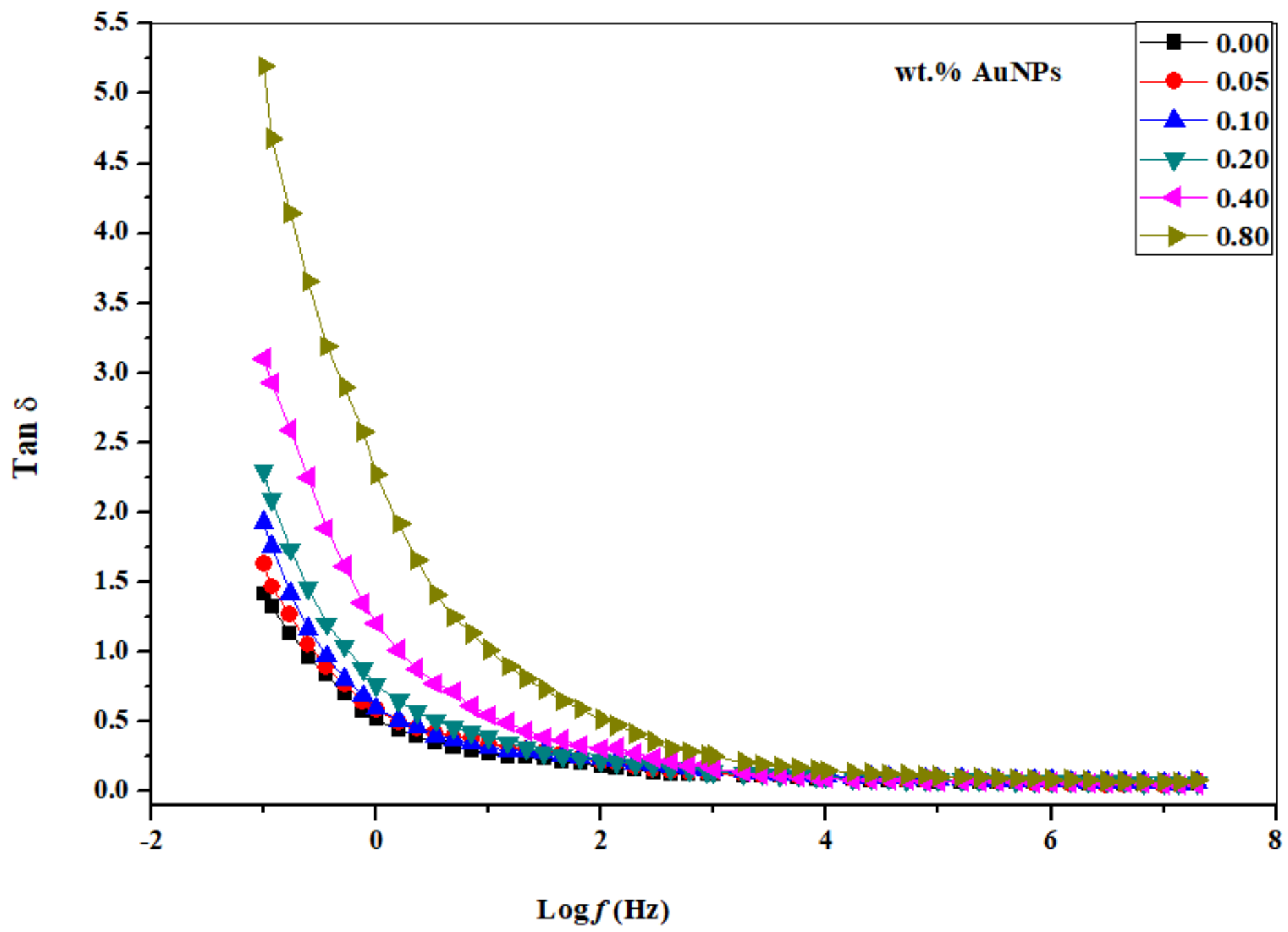

Figure 11

Variation of loss tangent ( $\tan \delta$ ) with respect to frequency of nanocomposites samples at room temperatures.

\section{Supplementary Files}

This is a list of supplementary files associated with this preprint. Click to download.

- Scheme1.png 